\title{
Small-scale environmental enrichment and exercise enhance learning and spatial memory of Carassius auratus, and increase cell proliferation in the telencephalon: an exploratory study
}

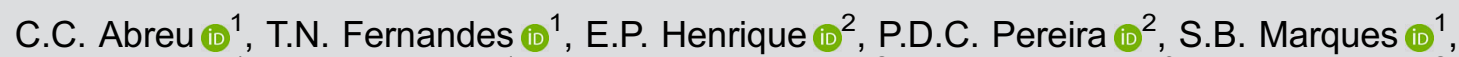 \\ S.L.S. Herdeiro ${ }^{1}$, F.R.R. Oliveira ${ }^{1}$, N.G.M. Magalhães $\mathbb{i i D}^{2}$, D.C. Anthony (iD) ${ }^{3}$, M.A.D. Melo (iD) ${ }^{2}$, \\ C. Guerreiro-Diniz (iD ${ }^{2}$, D.G. Diniz (ib ${ }^{1}$, and C.W. Picanço-Diniz (iD ${ }^{1}$ \\ ${ }^{1}$ Instituto de Ciências Biológicas, Hospital Universitário João de Barros Barreto, Laboratório de Neurodegeneração e Infecção, \\ Universidade Federal do Pará, Belém, PA, Brasil \\ ${ }^{2}$ Laboratório de Biologia Molecular e Neuroecologia, Instituto Federal de Educação Ciência e Tecnologia do Pará, \\ Bragança, PA, Brasil \\ ${ }^{3}$ University of Oxford, Department of Pharmacology, Mansfield Road, Oxford, United Kingdom
}

\begin{abstract}
Carassius auratus is a teleost fish that has been largely used in behavioral studies. However, little is known about potential environmental influences on its performance of learning and memory tasks. Here, we investigated this question in $C$. auratus, and searched for potential correlation between exercise and visuospatial enrichment with the total number of telencephalic glia and neurons. To that end, males and females were housed for 183 days in either an enriched (EE) or impoverished environment (IE) aquarium. EE contained toys, natural plants, and a 12-hour/day water stream for voluntary exercise, whereas the IE had none of the above. A third plus-maze aquarium was used for spatial and object recognition tests. Different visual clues in 2 of its 4 arms were used to guide fish to reach the criteria to complete the task. The test consisted of 30 sessions and was concluded when each animal performed three consecutive correct choices or seven alternated, each ten trials. Learning rates revealed significant differences between EE and IE fish. The optical fractionator was used to estimate the total number of telencephalic cells that were stained with cresyl violet. On average, the total number of cells in the subjects from EE was higher than those from subjects maintained in IE $(P=0.0202)$. We suggest that environmental enrichment significantly influenced goldfish spatial learning and memory abilities, and this may be associated with an increase in the total number of telencephalic cells.
\end{abstract}

Key words: Carassius auratus; Spatial learning and memory; Object recognition; Stereology; Telencephalon; Environmental enrichment

\section{Introduction}

The most primitive of the vertebrates, fish, have been described as possessing a poor differentiated telencephaIon, and limited learning and memory abilities (1). However, developmental, neuroanatomical, and functional studies suggest that the evolution of brain and behavior systems seems to be conserved in all vertebrates $(2,3)$, including teleost fishes $(4,5)$, where we can recognize many of the learning and memory abilities shared by mammals (6) and birds (7).

Object (what?), timing (when?), and placement recognition are well-established cognitive functions in birds and mammals that have been associated with hippocampal neurogenesis and gliogenesis $(8,9)$. Fish cell proliferation is affected by environmental changes but it has been suggested that fish may respond to the environment through processes that are not specific to behavioral change (10). Fish differ from birds and mammals $(11,12)$, and thus regional cell-proliferation specific responses in fishes should be interpreted under rigorous control of environmental variables to guarantee specificity of the environmental stimuli (13) (see (10) for recent review).

A few studies have investigated potential influences of environmental enrichment and exercise on spatial learning and memory performances in teleost fishes $(14,15)$ 
and none have investigated the effects of enriched environments on cell proliferation in the Carassius auratus telencephalon.

Although several $C$. auratus studies explored the remarkable parallelism between central nervous system morphology and functional organization of fishes, mammals, and birds (16-23), none of them investigated potential influences of environmental changes on learning and memory performances, and on telencephalic cell changes, using unbiased stereological methods. The present research addressed this question controlling for all other sources of potential confounding factors, including water temperature, $\mathrm{pH}, \mathrm{O}_{2}$ concentration, day-night cycle, noise level, and sex and number of individuals per volume of water in the aquarium.

The telencephalon was selected as the area of interest for counting cells because it has been described in $C$. auratus that its integrity, either medial $(24,25)$ or lateral $(18,22,23,26-28)$, are essential for spatial learning and memory.

\section{Material and Methods}

Female $C$. auratus were maintained in aquariums in accordance with the guidelines published by the National Institutes of Health (Guide for the Care and Use of Laboratory Animals). The experimental protocol was submitted and approved prior to study initiation by the Ethics Committee on Experimental Animal Research (Instituto de Ciências Biológicas, Universidade Federal do Pará, Brazil, CEPAE-UFPA: 0432015).

In the present research, we investigated the influence of small-scale environmental enrichment and exercise on spatial learning and memory in $C$. auratus using visual cued plus-maze apparatus (Figure $1 \mathrm{~A}-\mathrm{C}$ ) and evaluated the changes in the number of cells of the telencephalon. We used an unbiased quantitative stereological method for cell counts, the optical fractionator (29). To that end, we maintained 6-month-old fish for 183 days (6 months) in two distinct aquariums $(110 \times 55 \times 55 \mathrm{~cm})$ with a capacity of 332 liters. All aquariums contained biological filters (Sunsun Jp-025f $1600 \mathrm{~L} / \mathrm{h}$, Japan), internal circulation pump (Sunsun Wave Maker jvp-102b 5000 L/h 110V), thermometers (Aquarium digital thermometer), stones, ultra violet lamp, and 12-h light/dark cycle. On alternate days, oxygen, $\mathrm{pH}$, and temperature were measured and kept within the acceptable standards for this species (oxygen above $6.0 \mathrm{ppm}, \mathrm{pH}=7.0-7.6$, and temperature $22 \pm 1^{\circ} \mathrm{C}$ ). The mean values and standard errors were oxygen (EE: $6.95 \pm 1.8$; AP: $7.21 \pm 1.5$ ), pH (EE: $7.1 \pm$ 0.09 ; IE: $6.7 \pm 0.8$ ), and temperature $22.4 \pm 0.04^{\circ} \mathrm{C}$ ). The fish were fed twice daily with commercial feed (Sera Pond Bio Granulat, Brazil).

The enriched environment aquarium was equipped with a water pump that generated water flow for voluntary exercise, natural plants, and a resin boat to provide resting and shelter (Figure 1D). The impoverished aquarium did not contain any of these devices.

\section{Memory and learning test}

A plus-maze test apparatus (30) adapted to our experimental requirements (test aquarium) was used to assess spatial learning and memory performances. The plus-maze aquarium was maintained with similar water control parameters as previously described. Visual cues were placed on two arms of the apparatus, where squares indicated the right choice and circles the wrong one. The test was performed during three consecutive days; all fish performed 10 trials, reaching a total of 30 sessions by the end of the 3rd day, if the right choice did not occur before. All animals were adapted by entering into the apparatus $5 \mathrm{~min}$ before the beginning of the test. The trial started in the center of the apparatus, where fish remained for $1 \mathrm{~min}$, inside a rectangular glass lid. Then, the glass was removed, and the individual had free access to the 4 arms of the apparatus. As soon as the correct choice was selected,
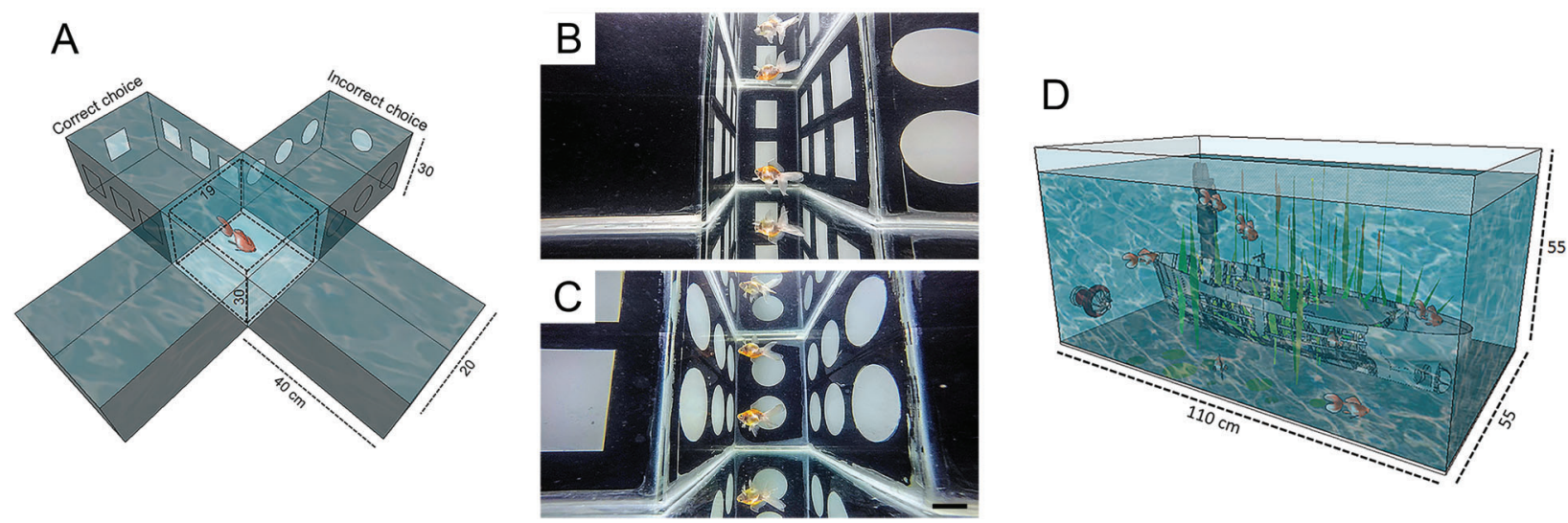

Figure 1. A, Illustration of the plus-maze apparatus. B, Correct choice in behavioral test. C, Incorrect choice in behavioral test. D, Illustration of enriched environment aquarium. Scale bar in $\mathbf{B}$ and $\mathbf{C}: 10 \mathrm{~cm}$. 
the individual was removed from the test aquarium and returned to its original aquarium. If the wrong choice was selected, we imposed a 1-min restriction on the swimming space, using a containment net at the bottom corners of the chosen arm, and then fish were transferred to their original aquarium. This restriction was systematically applied to all fish until the criteria to complete the task were reached, or the 30 sessions ended.

After behavioral tests, all animals were sacrificed using an overdose of $20 \%$ Avertin (tribromoethanol amyl alcohol, Sigma-Aldrich, USA) dissolved in the water of a small aquarium.

\section{Statistical analysis of behavioral data}

To assess learning rate and memory, we used KaplanMeier survival curve. A Kaplan-Meyer curve predicts time until an event and shows a series of declining horizontal steps, which provides, as a function of time, the true survival function for the sample under analysis. The main obstacle to the widespread use of survival analysis (such as in the present research) is the word "survival," which may lead to the misunderstanding that it can only be used for data related to death or failure (40). In the present work, we have made the following question: How long does it take for a fish to learn and remember the correct arm? (time for the occurrence of the correct choice versus wrong choice). Here, the event was reaching the learning and memory criteria as a function of the progression of the training session (time). In our sample, every time an individual achieved three consecutive correct choices, or
7 correct choices alternated with wrong ones, in a total of 10 trials, a decrease in the survival curve occurred (curve stepdown). Thus, the Kaplan-Meier survival curve in the present work illustrated the probability of the correct choice being made as a function of the number of training sessions. Because the EE individuals met the criteria faster than those maintained in an IE, their KaplanMeier curve showed a lower survival rate. The log-rank test was used to compare the two curves for significant differences.

\section{Fixation and histological procedures}

After craniotomy, brain tissue was fixed by immersion in buffered $10 \%$ formalin. Figure 2 illustrates a $C$. auratus specimen and its dissected brain in the dorsal, ventral, and lateral views. After 7 days, the brain was dissected and cut using a vibratome. Serial sections $70 \mu \mathrm{m}$ thick were collected and 1:3 anatomical series were kept in the same fixative and maintained at $4^{\circ} \mathrm{C}$ in the refrigerator. They were then mounted in gelatinized glass slides, airdried and stained with cresyl violet. After that, they were dehydrated, cleared, and mounted with DPX Mountant and cover-slipped (Sigma-Aldrich).

\section{Photomicrography}

Digital photomicrographs were taken with a digital camera (Microfire, Optronics, USA) attached to a Nikon microscope (Optiphot-2, USA). The levels of brightness and contrast applied to the entire image were adjusted with Adobe Photoshop CC 2018 (USA).

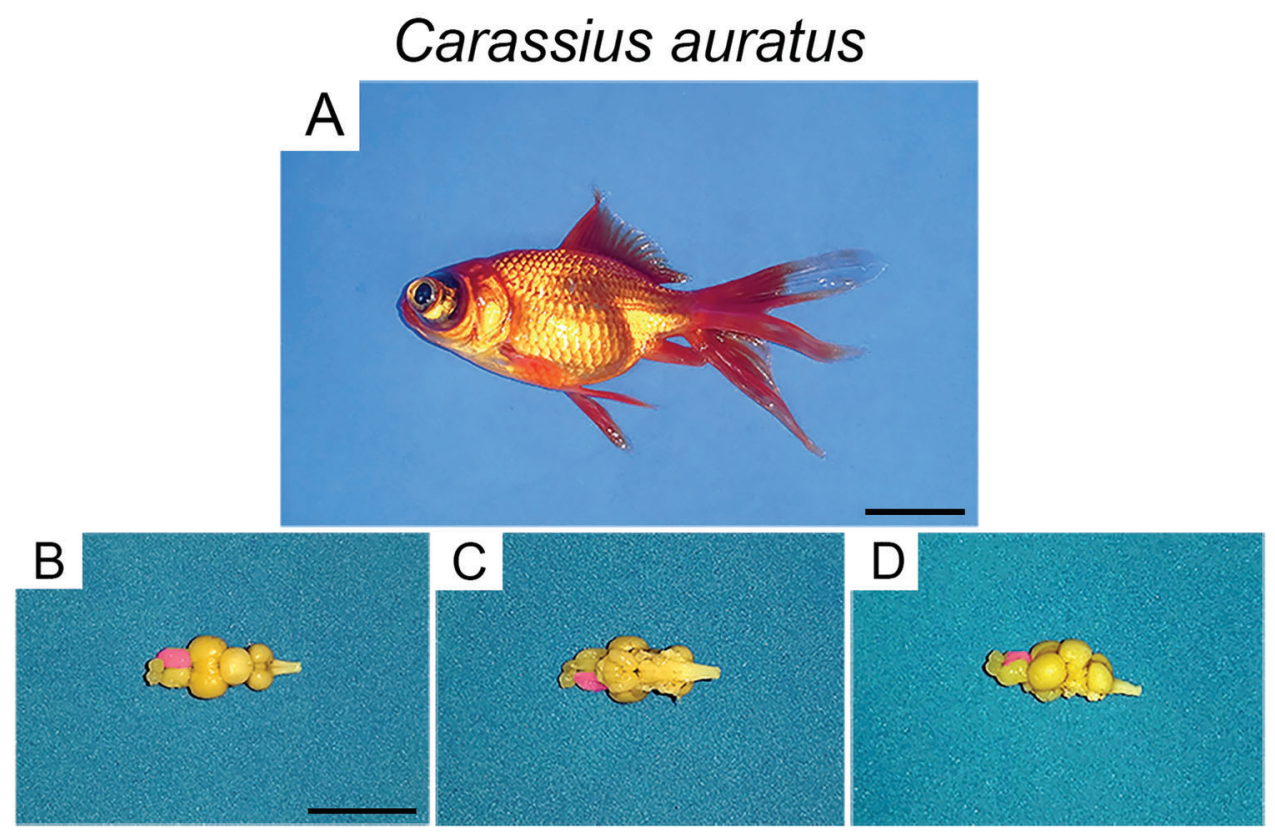

Figure 2. A, Carassius auratus. Dissected brain in dorsal view (B), ventral view (C), and lateral view (D). The right hemisphere is shown in pink. Scales: A: $2 \mathrm{~cm}$; B, C, and D: $1 \mathrm{~cm}$. 


\section{Stereology}

To investigate the influence of the environment on the total telencephalic cell numbers (neurons and glia), we compared the stereological estimates of the total number of cell counts from 7 individuals of each group, using the optical fractionator (29). The stereological analysis requires the correct identification of the region of interest. The telencephalon of $C$. auratus shows, as all other teleost fishes so far investigated, two solid hemispheres composed of several nuclear masses separated by a common ventricle (31). The boundaries of the telencephalon are readily recognized in Nissl-stained sections of teleost fishes (31). To count cells, we used the systematic and random distribution of counting blocks in telencephalic parasagittal sections. This sampling is a key step since it is not possible to count all cells within the region of interest. To overcome this dilemma and obtain estimates close to the real values, the stereological procedure requires the use of systematic and random data collection. This alternative ensures adequate estimation of the total number of cells from the number of cells detected in each sampled counting box and in the sample probability (32). However, to minimize methodological errors we needed to select grid and counting box dimensions that generated, after counting procedures, a Scheaffer coefficient of error smaller than 0.05. Methodological errors $\leqslant 0.05$ contribute little to the observed group variance. To fulfill this criterion, a pilot study was carried out where we tested different grid and counting boxes dimensions for the telencephalic sections of $C$. auratus, and counted cells, until we found appropriate coefficient of errors, increasing precision of the estimate.

\section{Results}

All animals were weighed before sacrifice and had the same age at sacrifice. The two-tailed $t$-test to detect potential differences in the body weight of the two experimental groups did not show any significant difference $(E E=22.04 \pm 2.49$ vs $\mathrm{IE}=21.0 \pm 1.44, \mathrm{t}=-0.98, \mathrm{P}=0.35)$.

\section{Area of interest, grid, and counting boxes}

Figure 3 shows low- and high-power photomicrographs of parasagittal Nissl-stained sections of the telencephalon of $C$. auratus maintained in enriched $(A-D)$ and impoverished $(\mathrm{E}-\mathrm{H})$ environments. To count cells, we did not distinguish between glia and neurons. Figure 4 indicates the right telencephalic hemisphere, a parasagittal cresyl violet-stained section taken from the level illustrated by the dotted line over the pink hemisphere, and the systematic and random sampling approach for counting cells. Arrows under the stained section indicate anatomical references. Counting boxes are placed in the corner of each square of the chosen grid using Stereo Investigator software (MBF Bioscience, USA). The colored lines in counting boxes indicated prohibited (red) and allowed (green) counting lines.

Table 1 shows the stereological parameters selected to minimize potential methodological errors and Tables 2 and 3 show the estimates of the total number of glial and neuronal cells of $C$. auratus maintained in $E E$ and IE aquariums. Compared with the total number of estimated cells on the telencephalon of fish maintained on the IE aquarium, on average, the total number of cells of individuals from $E E$ was significantly higher (means $\pm S D$, $\mathrm{EE}=1.12 \times 10^{6} \pm 0.23 \times 10^{6}$ vs $\mathrm{IE}=0.86 \times 10^{6} \pm 0.16 \times 10^{6}$; two-tailed $t$-test, $\mathrm{t}=2.3, \mathrm{P}=0.04$ ).

\section{Spatial learning and memory}

Figure 5 is a graphic representation of the learning rates and the total number of telencephalic cells of the two groups. Compared to performances of fish maintained in the IE aquarium, subjects from the EE learned faster how
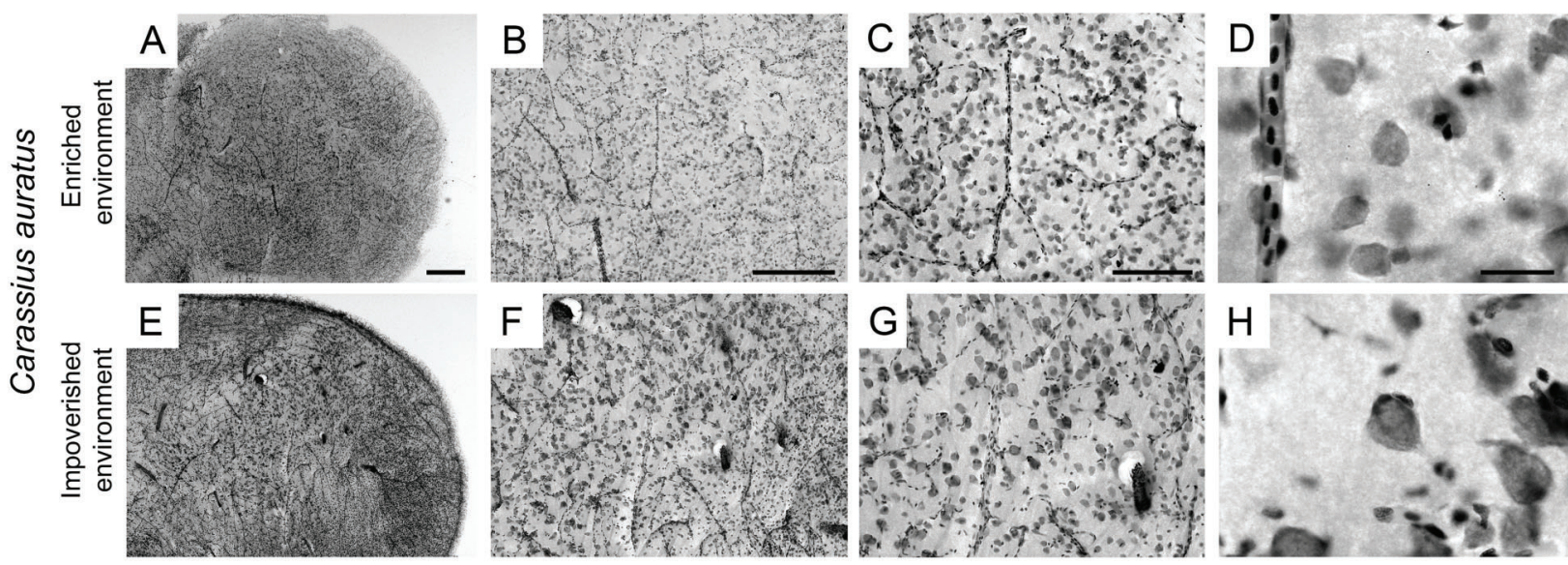

Figure 3. Photomicrographs of regions of interest (telencephalon) of $C$. auratus from enriched environment and impoverished environment, stained with cresyl violet dye. Magnification and bar size: A and E: 4x $(500 \mu \mathrm{m})$, B and F: 10x (250 $\mu \mathrm{m}), \mathbf{C}$ and G: 20x $(250 \mu \mathrm{m})$, D and H: $100 \times(25 \mu \mathrm{m})$. 

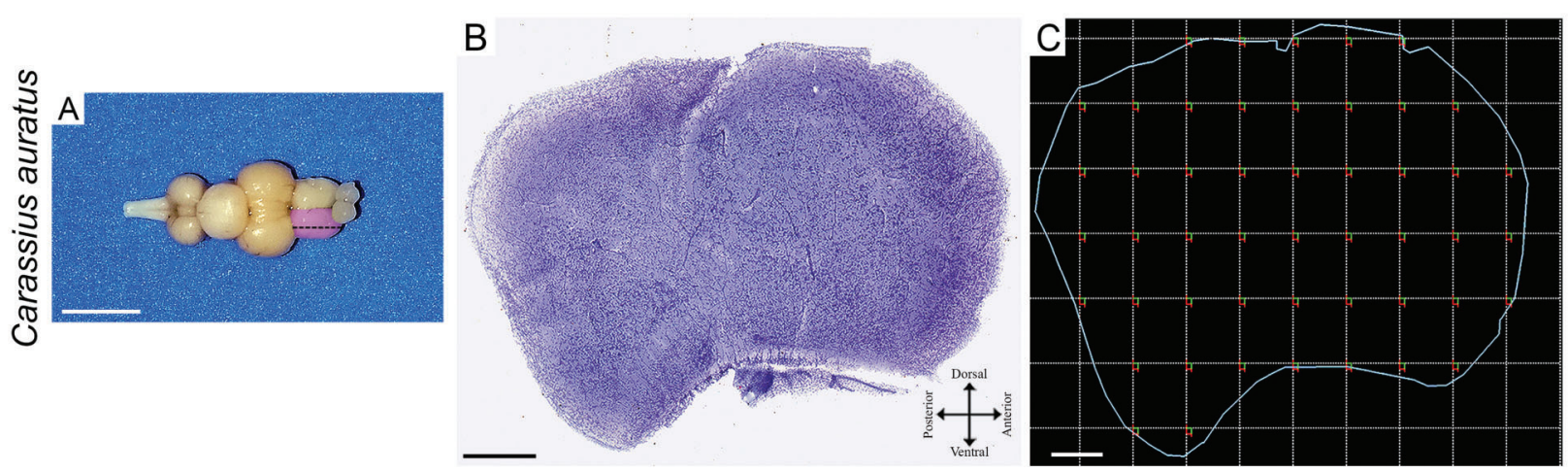

Figure 4. A, Photograph of the whole brain of $C$. auratus. The pink region indicates the right telencephalon, and the dotted line the location of the histological cut. B, Histological section of the telencephalon stained with cresyl violet showing how it was disposed on a slide. C, Outline of the systematic and random distribution of count boxes in the telencephalon region (blue line), demonstrating the layout and relative dimensions of the counting boxes (squares). The green edges of the count boxes demonstrate the limits of cell counting in the planes and the red edges the excluded plane limits. The width and length of the boxes is $30 \mu \mathrm{m}$ and the grid (in dotted lines) is $320 \mu \mathrm{m}$. Scale bars in A: $5 \mathrm{~mm}$; B: $400 \mu \mathrm{m}$; C: $320 \mu \mathrm{m}$.

Table 1. Stereological parameters for counting telencephalic cells of $C$. auratus maintained either in an environmentally enriched (EE) or impoverished (IE) aquarium.

\begin{tabular}{lcccc}
\hline $\begin{array}{l}\text { Carassius } \\
\text { auratus }\end{array}$ & $\begin{array}{c}\text { Thickness } \\
(\mu \mathrm{m})\end{array}$ & $\begin{array}{c}\text { Volume } \\
\left(\mathrm{mm}^{3}\right)\end{array}$ & $\begin{array}{c}\text { Total of } \\
\text { probes }\end{array}$ & $\begin{array}{c}\text { Counting boxes } \\
\text { total }\end{array}$ \\
\hline EE 02 & 13.3 & 5.78 & 8 & 277 \\
EE 03 & 18.3 & 4.14 & 7 & 199 \\
EE 04 & 17.6 & 5.67 & 7 & 278 \\
EE 05 & 16.3 & 5.50 & 8 & 266 \\
EE 08 & 16.2 & 5.52 & 8 & 272 \\
EE 11 & 14.3 & 7.32 & 8 & 359 \\
EE 12 & 16.3 & 6.48 & 7 & 316 \\
Mean & 16.04 & 5.77 & 7.67 & \\
SE & & 0.37 & & \\
IE 01 & 18.0 & 5.30 & 6 & 256 \\
IE 02 & 16.0 & 8.93 & 8 & 431 \\
IE 06 & 13.4 & 5.01 & 7 & 242 \\
IE 07 & 14.0 & 6.40 & 8 & 315 \\
IE 08 & 14.7 & 7.32 & 8 & 353 \\
IE 04 & 16.2 & 5.02 & 7 & 242 \\
IE 09 & 17.1 & 8.00 & 8 & 388 \\
Mean & 15.63 & 6.57 & 7.33 & \\
SE & & 0.59 & & \\
\hline
\end{tabular}

Dimensions: box size $30 \times 30 \mu \mathrm{m}$, grid $320 \times 320 \mu \mathrm{m}$, dissector height $10 \mu \mathrm{m}$, and interval between sections 1:3.

to locate and remember the correct arm to be explored (log-rank test, $\mathrm{P}=0.0354$ ). Compared with individuals from $\mathrm{IE}$, the total number of telencephalic cells was significantly higher in fishes from $E E$ (two-tailed $t$-test, $P=0.04$; see Tables 2 and 3 for details).

\section{Discussion}

Evidence suggests that $C$. auratus can navigate on the basis of allocentric maps and this is dependent on the integrity of the telencephalon $(18,22,23,26-28)$. In the 
Table 2. Estimation of the total number of glial and neuronal cells with their coefficient error for the Carassius auratus telencephalon of the enriched environment.

\begin{tabular}{lccc}
\hline Carassius auratus & Total cells & Thickness $(\mu \mathrm{m})$ & Scheaffer CE \\
\hline EE 02 & 864,690 & 13.3 & 0.045 \\
EE 03 & 995,356 & 18.3 & 0.060 \\
EE 04 & 983,777 & 17.6 & 0.049 \\
EE 05 & $1,103,967$ & 16.3 & 0.048 \\
EE 08 & $1,015,602$ & 16.2 & 0.056 \\
EE 11 & $1,380,236$ & 14.3 & 0.045 \\
EE 12 & $1,513,172$ & 16.3 & 0.050 \\
Mean & $1,122,400$ & 16.0 & 0.050 \\
SD & 235,477 & & \\
CV & 0.2098 & & \\
CV & & \\
CE & & & \\
CE $^{2} / C V^{2}$ & 0.044 & & \\
CV $^{2}-C^{2}$ & 0.0025 & & \\
CVB $^{2}(\%)$ & 0.057 & & \\
\hline
\end{tabular}

EE: enriched environment; $C E$ : coefficient error; CV: coefficient of variation; CVB: coefficient of biological variation; $\mathrm{CVB}^{2}=\mathrm{CV}^{2}-\mathrm{CE}^{2}$.

Table 3. Estimation of the total number of glial and neuronal cells with their coefficient error for the Carassius auratus telencephalon of the impoverished environment.

\begin{tabular}{lccc}
\hline Carassius auratus & Total cells & Thickness $(\mu \mathrm{m})$ & Scheaffer CE \\
\hline IE 01 & 723,446 & 18.0 & 0.057 \\
IE 02 & 803,130 & 16.0 & 0.048 \\
IE 06 & 708,942 & 13.4 & 0.052 \\
IE 07 & $1,075,795$ & 14.0 & 0.052 \\
IE 08 & $1,096,997$ & 14.7 & 0.045 \\
IE 04 & 661,802 & 16.2 & 0.050 \\
IE 09 & 975,856 & 17.1 & 0.045 \\
Mean & 863,709 & 15.6 & 0.050 \\
SD & 169,049 & & \\
CV & 0.1957 & & \\
CV & 0.038 & & \\
CE & & & \\
CE $^{2} /$ CV $^{2}$ & 0.0025 & & \\
CV $^{2}-C^{2}$ & 0.06 & & \\
CVB $^{2}(\%)$ & 0.035 & & \\
\hline
\end{tabular}

IE: impoverished environment; CE: coefficient error; CV: coefficient of variation; CVB: coefficient of biological variation; $\mathrm{CVB}^{2}=\mathrm{CV}^{2}-\mathrm{CE}^{2}$.

present study, we found that compared with individuals maintained for 6 months in an IE aquarium, the total number of cells in the telencephalon of individuals maintained in an EE aquarium was increased, and this coincided with a better performance on the learning and memory plus-maze task. These findings are in line with previous evidence in mammals and birds demonstrating a significant increase of the total number of neurons and glial cells on the hippocampus of a variety species so far investigated $(8,9)$. 

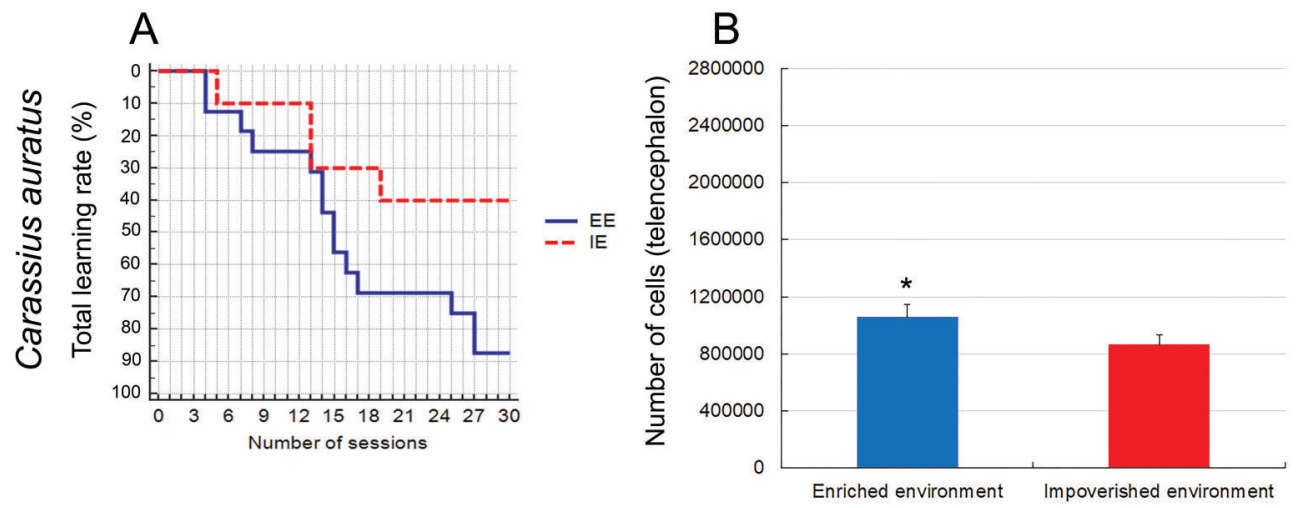

Figure 5. A, Graphical representation of the behavioral test of enriched (blue) and impoverished (red) groups of $C$. auratus (KaplanMeyer analysis, $P=0.0354$ ) in all animals. B, Estimated number of glial and neuronal cells in the telencephalon using the optical fractionator in the two experimental groups $(P=0.0404)$. Data are reported as mean $\pm S D$. ${ }^{*} \mathrm{P}<0.05$ (two-tailed $t$-test). IE: impoverished environment; EE: enriched environment.

Different from the endothermal mammals and birds, ectotherms do exhibit cell proliferation due to nonspecific influences of the environment, which may act indirectly through changes in body temperature $(33,34)$, sex $(35)$, age (36), and somatic and neural injuries followed by regeneration (37). These effects may influence cell counts (10). To minimize these influences, in the present study, we maintained experimental variables under control, including water temperature, $\mathrm{pH}, \mathrm{O}_{2}$ concentration, day-night cycle, noise level, sex, age, and number of individuals per volume of water, in both environments. Thus, we expected that the significant differences found were rather specific.

As previously mentioned, the number of brain cells in teleost fishes increases with age, body weight, and body length throughout life, but studies on potential influences of environmental enrichment are scarce. Studies on telencephalic cell proliferation in the forebrain of zebrafish (Danio rerio) maintained in an enriched aquarium with artificial plants, demonstrated higher numbers of cells immunolabeled for proliferating cell nuclear antigen, suggesting that environmental changes may alter the cell cycle of zebrafish (38). Similarly, previous results in Brachyhypopomus gauderio, using bromodeoxyuridine as the cell marker, demonstrated an increase in cell proliferation across the brain in individuals maintained in a wild environment compared with the individuals maintained in captivity (13). Finally, in the Salmoniformes species Oncorhynchus kisutch and in the Salmo salar species subjected to environmental complex stimuli, an increase in BrdU (39) and NeuroD1 mRNA (15), respectively, was found in both dorsomedial and dorsolateral telencephalic regions compared with individuals maintained in a simple environmental structure.
Although we did not investigate the subjacent mechanisms in the present study, we expanded previous observations to $C$. auratus, demonstrating that the cell cycle of its telencephalon was also altered by small-scale physical environmental enrichment, and that these changes coincided with an enhanced performance on spatial learning and memory.

To quantify changes in telencephalic number of cells, we applied the optical fractionator, an accurate method of quantification combining properties of an optical dissector and the fractionator that has been used in a variety of studies to determine cell numbers in multiple brain regions. The optical fractionator is unaffected by histological changes or shrinkage, an issue of importance when performing comparative analysis between experimental groups $(37,39$, 40). The main variability in the present analysis was biological variability (91-92\% of the total variation), with CE/ $\mathrm{CV}<0.5$. As a result, possible variations associated with non-biological sources were reduced to acceptable levels.

\section{Acknowledgments}

We acknowledge the following for financial support: Fundação de Amparo à Pesquisa do Pará (FADESP)/PróReitoria de Pesquisa e Pós-Graduação da Universidade Federal do Pará (PROPESP) Edital 02-2018-PIAPA; Coordenação de Aperfeiçoamento de Pessoal de Nível Superior (CAPES) - Pró-Amazônia, Grant No. 3311/2013; Brazilian Research Council (CNPq) Grant Nos: 307749/ 2004-5 and 471077/2007-0 to C.W. Picanço-Diniz; Fundação Amazônia de Amparo a Estudos e Pesquisas do Pará (FAPESPA) ICAAF No. 039/2017. 


\section{References}

1. Salas C, Broglio C, Durán E, Gómez A, Ocaña FM, JiménezMoya $F$, et al. Neuropsychology of learning and memory in teleost fish. Zebrafish 2006; 3: 157-171, doi: 10.1089/zeb. 2006.3.157.

2. Allen TA, Fortin NJ. The evolution of episodic memory. Proc Natl Acad Sci USA 2013; 110: 10379-10386, doi: 10.1073/ pnas.1301199110.

3. Reiter S, Liaw HP, Yamawaki TM, Naumann RK, Laurent G. On the value of reptilian brains to map the evolution of the hippocampal formation. Brain Behav Evol 2017; 90: 41-52, doi: $10.1159 / 000478693$.

4. Murray EA, Wise SP, Graham KS. Representational specializations of the hippocampus in phylogenetic perspective. Neurosci Lett 2018; 680: 4-12, doi: 10.1016/j.neulet.2017. 04.065.

5. Gerlai R. Zebrafish and relational memory: Could a simple fish be useful for the analysis of biological mechanisms of complex vertebrate learning? Behav Processes 2017; 141: 242-250, doi: 10.1016/j.beproc.2017.01.016.

6. Ranganath C. Time, memory, and the legacy of Howard Eichenbaum. Hippocampus 2018, doi: 10.1002/hipo.23007.

7. Herold C, Coppola VJ, Bingman VP. The maturation of research into the avian hippocampal formation: Recent discoveries from one of the nature's foremost navigators. Hippocampus 2015; 25: 1193-1211, doi: 10.1002/hipo.22 463.

8. Sherry DF, MacDougall-Shackleton SA. Seasonal change in the avian hippocampus. Front Neuroendocrinol 2015; 37 : 158-167, doi: 10.1016/j.yfrne.2014.11.008.

9. Cooper C, Moon HY, van Praag H. On the Run for Hippocampal Plasticity. Cold Spring Harb Perspect Med 2018; 8: pii: a029736, doi: 10.1101/cshperspect.a029736.

10. Dunlap KD. Fish neurogenesis in context: assessing environmental influences on brain plasticity within a highly labile physiology and morphology. Brain Behav Evol 2016; 87: 156-66, doi: 10.1159/000446907.

11. Opendak M, Gould E. Adult neurogenesis: a substrate for experience-dependent change. Trends Cogn Sci 2015; 19: 151-161, doi: 10.1016/j.tics.2015.01.001.

12. Barker JM, Boonstra R, Wojtowicz JM. From pattern to purpose: how comparative studies contribute to understanding the function of adult neurogenesis. Eur $\mathrm{J}$ Neurosci 2011; 34: 963-977, doi: 10.1111/j.1460-9568.2011.07823.x.

13. Dunlap KD, Silva AC, Chung M. Environmental complexity, seasonality and brain cell proliferation in a weakly electric fish, Brachyhypopomus gauderio. J Exp Biol 2011; 214: 794-805, doi: 10.1242/jeb.051037.

14. Luchiari AC, Chacon DM. Physical exercise improves learning in zebrafish, Danio rerio. Behav Processes 2013; 100: 44-47, doi: 10.1016/j.beproc.2013.07.020.

15. Salvanes AG, Moberg O, Ebbesson LO, Nilsen TO, Jensen $\mathrm{KH}$, Braithwaite VA. Environmental enrichment promotes neural plasticity and cognitive ability in fish. Proc Biol Sci 2013; 280: 20131331, doi: 10.1098/rspb.2013.1331.

16. Ocaña FM, Uceda S, Arias JL, Salas C, Rodríguez F. Dynamics of goldfish subregional hippocampal pallium activity throughout spatial memory formation. Brain Behav Evol 2017; 9: 154-170, doi: 10.1159/000478843.
17. Rodríguez-Expósito B, Gómez A, Martín-Monzón I, Reiriz M, Rodríguez F, Salas C. Goldfish hippocampal pallium is essential to associate temporally discontiguous events. Neurobiol Learn Mem 2017; 139: 128-134, doi: 10.1016/ j.nlm.2017.01.002.

18. Broglio C, Rodríguez F, Gómez A, Arias JL, Salas C. Selective involvement of the goldfish lateral pallium in spatial memory. Behav Brain Res 2010; 210: 191-201, doi: 10.1016/j.bbr.2010.02.031.

19. Portavella M, Vargas JP, Torres B, Salas C. The effects of telencephalic pallial lesions on spatial, temporal, and emotional learning in goldfish. Brain Res Bull 2002; 57: 397-399, doi: 10.1016/S0361-9230(01)00699-2.

20. Vargas JP, Bingman VP, Portavella M, López JC. Telencephalon and geometric space in goldfish. Eur J Neurosci 2006; 24: 2870-2878, doi: 10.1111/j.1460-9568.2006.05174.x.

21. Gómez Y, Vargas JP, Portavella M, López JC. Spatial learning and goldfish telencephalon NMDA receptors. Neurobiol Learn Mem 2006; 85: 252-262, doi: 10.1016/j.nlm.2005. 11.006.

22. López JC, Bingman VP, Rodríguez F, Gómez Y, Salas C. Dissociation of place and cue learning by telencephalic ablation in goldfish. Behav Neurosci 2000; 114: 687-699, doi: 10.1037/0735-7044.114.4.687.

23. Salas C, Rodríguez F, Vargas JP, Durán E, Torres B. Spatial learning and memory deficits after telencephalic ablation in goldfish trained in place and turn maze procedures. Behav Neurosci 1996; 110: 965-980, doi: 10.1037/0735-7044.110. 5.965.

24. Saito K, Watanabe S. Spatial learning deficits after the development of dorsomedial telencephalon lesions in goldfish. Neuroreport 2004; 15: 2695-2699, doi: 10.1097/0000 1756-200405190-00006.

25. Saito K, Watanabe S. Deficits in acquisition of spatial learning after dorsomedial telencephalon lesions in goldfish. Behav Brain Res 2006; 172: 187-194, doi: 10.1016/j.bbr. 2006.04.014.

26. Durán E, Ocańa FM, Gómez A, Jiménez-Moya F, Broglio C, Rodríguez $\mathrm{F}$, et al. Telencephalon ablation impairs goldfish allocentric spatial learning in a "hole-board" task. Acta Neurobiol Exp (Wars) 2008; 68: 519-525.

27. Rodriguez F, Lopez JC, Vargas JP, Gomez Y, Broglio C, Salas C. Conservation of spatial memory function in the pallial forebrain of reptiles and ray-finned fishes. $J$ Neurosci 2002; 22: 2894-2903, doi: 10.1523/JNEUROSCI.22-0702894.2002.

28. Salas C, Broglio C, Rodríguez F, López JC, Portavella M, Torres B. Telencephalic ablation in goldfish impairs performance in a 'spatial constancy' problem but not in a cued one. Behav Brain Res 1996; 79: 193-200, doi: 10.1016/ 0166-4328(96)00014-9.

29. West MJ. Stereological methods for estimating the total number of neurons and synapses: issues of precision and bias. Trends Neurosci 1999; 22: 51-61, doi: 10.1016/S01662236(98)01362-9.

30. Sison M, Gerlai R. Associative learning in zebrafish (Danio rerio) in the plus maze. Behav Brain Res 2010; 207: 99-104, doi: 10.1016/j.bbr.2009.09.043. 
31. Ito $\mathrm{H}$, Yamamoto $\mathrm{N}$. Non-laminar cerebral cortex in teleost fishes? Biol Lett 2009; 5: 117-121, doi: 10.1098/rsbl.2008. 0397.

32. Schmitz C, Hof PR. Design-based stereology in neuroscience. Neuroscience 2005; 130: 813-831, doi: 10.1016/ j.neuroscience.2004.08.050.

33. Rieder CL, Cole RW. Cold-shock and the Mammalian cell cycle. Cell Cycle 2002; 1: 169-175, doi: 10.4161/cc.1.3.119.

34. Radmilovich M, Fernández A, Trujillo-Cenóz O. Environment temperature affects cell proliferation in the spinal cord and brain of juvenile turtles. J Exp Biol 2003; 206: 3085-3093, doi: 10.1242/jeb.00515.

35. Perry AN, Grober MS. A model for social control of sex change: interactions of behavior, neuropeptides, glucocorticoids, and sex steroids. Horm Behav 2003; 43: 31-38, doi: 10.1016/S0018-506X(02)00036-3.

36. Zikopoulos B, Kentouri M, Dermon CR. Proliferation zones in the adult brain of a sequential hermaphrodite teleost species (Sparus aurata). Brain Behav Evol 2000; 56: 310 322, doi: $10.1159 / 000047215$.
37. Ilieş I, Sîrbulescu RF, GK Z. Indeterminate body growth and lack of dynamics, neurogenesis, apoptosis, and gliosis in the adult brain: a novel teleost fish model of negligible senescence. gonadal decline in the brown ghost knifefish (Apteronotus leptorhynchus), an organism exhibiting negligible brain senescence. Can J Zool 2014; 92: 947-953, doi: 10.1139/cjz-2014-0109.

38. von Krogh K, Sørensen C, Nilsson GE, Øverli Ø. Forebrain cell proliferation, behavior, and physiology of zebrafish, Danio rerio, kept in enriched or barren environments. Physiol Behav 2010; 101: 32-39, doi: 10.1016/j.physbeh. 2010.04.003.

39. Lema SC, Hodges MJ, Marchetti MP, Nevitt GA. Proliferation zones in the salmon telencephalon and evidence for environmental influence on proliferation rate. Comp Biochem Physiol A Mol Integr Physiol 2005; 1413: 327-335, doi: 10. 1016/j.cbpb.2005.06.003.

40. In J, Lee DK. Survival analysis: Part I - analysis of time-toevent. Korean J Anesthesiol 2018; 71: 182-19, doi: 10.4097/ kja.d.18.00067. 